\title{
Event-triggered Control for Vehicle Platooning
}

\author{
Steffen Linsenmayer ${ }^{1}$ and Dimos V. Dimarogonas ${ }^{2}$
}

\begin{abstract}
This paper presents a method to control vehicular platoons in an event-triggered fashion. Therefore, every vehicle broadcasts its position and velocity information only at discrete event times. These events are determined by a trigger rule only depending on the agents state and on time. Two control architectures are considered. The first one, called symmetric bidirectional, uses information of the front and back neighbor in the control law. The architecture is analyzed with a linear controller and it is shown that the state error converges to an adjustable region around the origin. In earlier work it is suggested to use a nonlinear controller if solely information from the front neighbor is available. Thus, a nonlinear eventtriggered predecessor-following control is developed and analyzed additionally. Not only bounds are given for the state of each vehicle, but it is also shown that the converging input converging state property holds. In both cases we guarantee the existence of a lower bound on the inter-event times. The benefits of both strategies are verified in numerical simulations.
\end{abstract}

\section{INTRODUCTION}

An important idea to prevent traffic congestion and increase road safety is to run a big group of vehicles in a platoon with small gaps between the vehicles. Two decentralized control architectures only depending on nearest neighbor interaction are stated in [1] to control such a platoon. Since the paper gives a detailed analysis of linear and nonlinear controllers regarding stability and robustness this is the motivating paper on which we build up our work. A possible extension to the configuration in [1] is to assume the platoon being equipped with a communication network and therefore the control should be adapted to keep the network load low while preserving the performance of the controller. To reduce the network load we try to combine the work on vehicular platoons with current work on event-triggered control of multi-agent systems. Since the vehicles are modeled with double-integrator dynamics in this paper especially work on multi-agent systems with double-integrator dynamics, like in [2] seems to be relevant.

Control of vehicular platoons is a well known problem. In [3] the current progress on longitudinal control problems in platoons of vehicles is summarized with focus on good ways to model the system and find necessary sensors and actuators to design a controller. Much work has been done in the analysis of platoon stability, which is based on the string stability concept introduced in [4]. In [5] a controller for a detailed vehicle model is stated that guarantees vehicle

\footnotetext{
${ }^{1}$ Steffen Linsenmayer is with the Institute for Systems Theory and Automatic Control, University of Stuttgart, Germany steffen.linsenmayerdist. uni-stuttgart. de

${ }^{2}$ Dimos V. Dimarogonas is with the ACCESS Linnaeus Centre, School of Electrical Engineering, KTH Royal Institute of Technology, Stockholm, Sweden dimos@kth. se. His work has been supported by the Swedish Research Council (VR).
}

stability as well as platoon stability for different spacing strategies and only depends on relative information of front and back neighbor. Error amplification and disturbance propagation through the platoon are investigated in [6] and [7] for platoons with nearest neighbor interaction. [8] looks at string stability under communication constraints, assuming the platoon has a communication network. Assuming such a communication network, another interesting question that arises is the influence of the network topology on the platoon behavior. The work in [9] and [10] discusses this topic. In [1], two control architectures are investigated as linear and nonlinear controllers. The analysis combines analytic and numerical results on stability and robustness and therefore serves as a good basis to build our work on event-triggered control of vehicular platoons.

The idea of event-triggered control is that the controller is not updated continuously but only at certain times. In contrast to time-triggered control these discrete times are not known before, e.g., through a fixed trigger time, but the event times are determined by a trigger rule. Early work on event-triggered control introduced an event-based PID controller in [11]. Further work investigated event-based control for larger classes of systems. The work in [12] treats nonlinear systems being rendered input-to-state stable ([13]) by a given controller. It states a trigger law that guarantees asymptotic stability and guarantees that the time between two events is lower bounded. Enhancements based on this work lead to a Lyapunov framework that guarantees asymptotic stability and can be used to synthesize trigger laws in [14]. In [12] the possibility to apply event-triggered control to networked dynamic systems is mentioned. In these systems a big improvement is expected if every agent decides on its own when it broadcasts its state information, since then not only the control effort but also the network load is reduced. This concept is analyzed in [15] for a class of networked dynamic systems with controllers that fulfill a certain matching condition. In [16] the well known controller for the consensus problem from [17] is investigated in an event-triggered and self-triggered implementation. The work in [2] also focuses on the consensus problem not only in the single-integrator but also in the double-integrator case. In this work each agent only observes its own state and the trigger rule decides when to send the information to its neighbors. In this paper we fuse the design scheme of [2] with the control laws in [1] to derive event-triggering rules for platooning.

The following Section gives some necessary definitions and states the problem for the following two main parts. In Section III a linear event-triggered control in symmetric bidirectional architecture is analyzed, while a nonlinear 


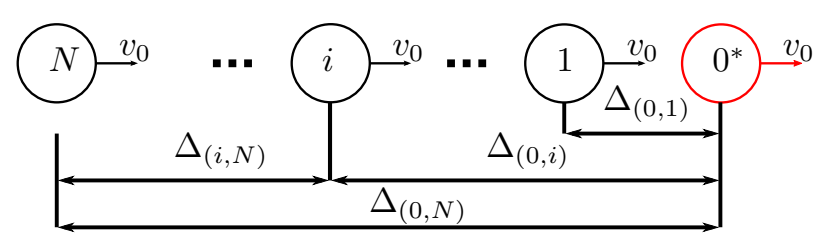

Fig. 1. Desired configuration with constant spacing and constant velocities with a fictitious leader agent $0^{*}$

predecessor-following control is investigated in Section IV. Section $\mathrm{V}$ gives some simulations verifying the theoretical results and showing the benefits of this work, ending in a conclusion in Section VI.

\section{DEFINITIONS AND PROBLEM STATEMENT}

Firstly we want to state some terminology from graph theory as well as concerning event-triggered control. Afterwards we state the problem being investigated in this paper.

\section{A. Definitions}

The graph class $\mathcal{G}=(\mathcal{V}, \mathcal{E})$ considered in this paper consists of a set of nodes $\mathcal{V}$, with $|\mathcal{V}|=N+N_{r}$, where $N$ is the number of real agents and $N_{r}$ is the number of fictitious reference agents, and edges $\mathcal{E}$. The Laplacian of a graph, i.e., $L(\mathcal{G}) \in \mathbb{R}^{|\mathcal{V}| \times|\mathcal{V}|}$ is defined as in [18], where many matrix properties and further definitions are stated. Furthermore if we eliminate column $k$ and row $k$ for each virtual reference agent $k$ we get the grounded Laplacian matrix $L_{g}(\mathcal{G}) \in \mathbb{R}^{N \times N}$, which is used in [9].

In our event-triggered control scheme every agent decides on its own at what time it transmits its current state to its neighbors. The discrete times when agent $i$ triggers its state information are denoted by $t_{k}^{i}$ with $k \in \mathbb{N}$ and the transmitted state at this time is $\hat{x}=x\left(t_{k}^{i}\right)$. One important property in event-triggered control is avoiding Zeno behavior. Zeno behavior occurs according to [19] when there is an infinite number of events triggered in finite time.

\section{B. Problem statement}

Every vehicle in the platoon is modeled as a fully actuated point mass moving in one dimension, i.e.,

$$
\ddot{p}_{i}=u_{i}, \quad i \in\{1, \ldots, N\} .
$$

The control objective is, as in [1], to keep a formation which is specified by a fictitious reference agent and positive constant gaps $\Delta_{(i-1, i)}$ between vehicles $i-1$ and $i$. The constant velocity trajectory of reference agent 0 is given as $p_{0}^{*}(t)=v_{0} t+p_{0,0}$ and only agent 1 knows this trajectory. Therefore the desired trajectory of agent $i$, i.e., $p_{i}^{*}(t)=$ $p_{0}^{*}(t)-\Delta_{(0, i)}=p_{0}^{*}(t)-\sum_{j=1}^{i} \Delta_{(j-1, j)}$ is not accessible to agent $i \in\{2, \ldots, N\}$. The desired configuration is illustrated in Fig. 1.

Furthermore [1] states two different control schemes. In the first one, called predecessor-following architecture, the control of each agent only depends on relative position and velocity measurements to its front neighbor and the desired gap, i.e., $\forall i \in\{1, \ldots, N\}$

$$
u_{i}=-f\left(p_{i}-p_{i-1}+\Delta_{(i-1, i)}\right)-g\left(\dot{p}_{i}-\dot{p}_{i-1}\right) .
$$

Agent 1 applies the same control law with the fictitious reference agent as its predecessor $p_{0}(t)=p_{0}^{*}(t)$.

The other control scheme is called symmetric bidirectional architecture and thus takes also into account relative position and velocity measurement to the back neighbor for all vehicles excluding the last one, since it has no following vehicle, i.e., $\forall i \in\{1, \ldots, N-1\}$

$$
\begin{aligned}
u_{i}= & -f\left(p_{i}-p_{i-1}+\Delta_{(i-1, i)}\right)-g\left(\dot{p}_{i}-\dot{p}_{i-1}\right) \\
& -f\left(p_{i}-p_{i+1}-\Delta_{(i, i+1)}\right)-g\left(\dot{p}_{i}-\dot{p}_{i+1}\right) \\
u_{N}= & -f\left(p_{N}-p_{N-1}+\Delta_{(N-1, N)}\right)-g\left(\dot{p}_{N}-\dot{p}_{N-1}\right) .
\end{aligned}
$$

In both cases we assume $f, g: \mathbb{R} \rightarrow \mathbb{R}$ to be odd and smooth enough to guarantee existence of a solution.

With continuous exchange of information these two control architectures are well studied in [1] according to stability and robustness. We try to investigate them now under eventtriggered control. The basic concept is that agent $i$ broadcasts its state information only when a trigger function $f_{i}(\cdot)$ reaches a specific value. To state the trigger function we introduce the transmitted errors for all $t: t_{k}^{i} \leq t<t_{k+1}^{i}$

$$
\begin{aligned}
e_{i}(t) & =\hat{p}_{i}+\left(t-t_{k}^{i}\right) \hat{\dot{p}}_{i}-p_{i}(t) \\
e_{d_{i}}(t) & =\hat{\dot{p}}_{i}-\dot{p}_{i}(t),
\end{aligned}
$$

where $\hat{p}_{i}=p_{i}\left(t_{k}^{i}\right)$ and $\hat{\dot{p}}_{i}=\dot{p}_{i}\left(t_{k}^{i}\right)$ are the last transmitted position and velocity values from agent $i$. From [2] we use the trigger condition $f_{i}(\cdot)>0$ and the trigger function

$$
f_{i}\left(t, e_{i}(t), e_{d_{i}}(t)\right)=\left\|\left[\begin{array}{c}
e_{i}(t) \\
e_{d_{i}}(t)
\end{array}\right]\right\|-\left(c_{0}+c_{1} e^{-\alpha t}\right) .
$$

This means that agent $i$ transmits its state to its neighbors when $f_{i}>0$, i.e., when $\left\|h_{i}(t)\right\|=\left\|\left[\begin{array}{ll}e_{i}(t) & e_{d_{i}}(t)\end{array}\right]^{T}\right\|>$ $c_{0}+c_{1} e^{-\alpha t}$. In the following Sections we analyze the behavior of the two controllers (2),(3) in an event-triggered implementation. The controller uses a first-order hold for the position and a zero-order hold for velocity information. In the case of a predecessor-following control architecture this leads to $\ddot{p}_{i}=-f\left(\hat{p}_{i}+\left(t-t_{k}^{i}\right) \hat{\dot{p}}_{i}-\hat{p}_{i-1}-\left(t-t_{k}^{i-1}\right) \hat{\dot{p}}_{i-1}+\right.$ $\left.\Delta_{(i-1, i)}\right)-g\left(\hat{\dot{p}}_{i}-\hat{\dot{p}}_{i-1}\right)$ for all $i=\{1, \ldots, N\}$. We define the state errors

$$
\tilde{p}_{i}(t)=p_{i}(t)-p_{i}^{*}(t), \dot{\tilde{p}}_{i}(t)=\dot{p}_{i}(t)-v_{0}
$$

and use (4), (6) to give the state error dynamics of the closed loop with event-triggered predecessor-following control architecture

$$
\begin{aligned}
\ddot{\tilde{p}}_{i}(t)= & -f\left(\tilde{p}_{i}(t)-\tilde{p}_{i-1}(t)+e_{i}(t)-e_{i-1}(t)\right) \\
& -g\left(\dot{\tilde{p}}_{i}(t)-\dot{\tilde{p}}_{i-1}(t)+e_{d_{i}}(t)-e_{d_{i-1}}(t)\right) .
\end{aligned}
$$


With the symmetric bidirectional control architecture the closed-loop system is

$$
\begin{aligned}
\ddot{\tilde{p}}_{i}(t)= & -f\left(\tilde{p}_{i}(t)-\tilde{p}_{i-1}(t)+e_{i}(t)-e_{i-1}(t)\right) \\
& -g\left(\dot{\tilde{p}}_{i}(t)-\dot{\tilde{p}}_{i-1}(t)+e_{d_{i}}(t)-e_{d_{i-1}}(t)\right) \\
& -f\left(\tilde{p}_{i}(t)-\tilde{p}_{i_{+1}}(t)+e_{i}(t)-e_{i+1}(t)\right) \\
& -g\left(\dot{\tilde{p}}_{i}(t)-\dot{\tilde{p}}_{i+1}(t)+e_{d_{i}}(t)-e_{d_{i+1}}(t)\right) \\
\ddot{\tilde{p}}_{N}(t)= & -f\left(\tilde{p}_{N}(t)-\tilde{p}_{N-1}(t)+e_{N}(t)-e_{N-1}(t)\right) \\
& -g\left(\dot{\tilde{p}}_{N}(t)-\tilde{\tilde{p}}_{N-1}(t)+e_{d_{N}}(t)-e_{d_{N-1}}(t)\right) .
\end{aligned}
$$

This is the basis for our analysis with trigger rule (5) regarding (asymptotic) bounds on the state errors while avoiding Zeno behavior.

\section{LINEAR EVENT-TRIGGERED CONTROL IN SYMMETRIC BIDIRECTIONAL ARCHITECTURE}

Firstly the event-triggered symmetric bidirectional control architecture with $f$ and $g$ being linear is investigated, i.e., $f(z)=k z, g(z)=b z$ with $k, b>0$. Using (8) and the stack vectors

$$
\begin{aligned}
x(t) & =\left[\begin{array}{lllll}
\tilde{p}_{1}(t) & \dot{\tilde{p}}_{1}(t) & \ldots & \tilde{p}_{N}(t) & \dot{\tilde{p}}_{N}(t)
\end{array}\right]^{T}, \\
e(t) & =\left[\begin{array}{lllll}
e_{1}(t) & e_{d_{1}}(t) & \ldots & e_{N}(t) & e_{d_{N}}(t)
\end{array}\right]^{T} \in \mathbb{R}^{2 N}
\end{aligned}
$$

it is possible to write the closed loop dynamics in state space form $\dot{x}(t)=A_{S B} x(t)+B_{S B} e(t), x(0)=x_{0} \in \mathbb{R}^{2 N}$. The matrices $A_{S B}$ and $B_{S B}$ can be computed as

$$
\begin{aligned}
& A_{S B}=I_{N} \otimes A_{1}+L_{g}\left(\mathcal{G}_{S B}\right) \otimes A_{2} \in \mathbb{R}^{2 N \times 2 N}, \\
& B_{S B}=L_{g}\left(\mathcal{G}_{S B}\right) \otimes A_{2} \in \mathbb{R}^{2 N \times 2 N},
\end{aligned}
$$

with $A_{1}=\left[\begin{array}{ll}0 & 1 \\ 0 & 0\end{array}\right], A_{2}=\left[\begin{array}{cc}0 & 0 \\ -k & -b\end{array}\right]$ and

$$
L_{g}\left(\mathcal{G}_{S B}\right)=\left[\begin{array}{cccc}
2 & -1 & & 0 \\
-1 & \ddots & \ddots & \\
& \ddots & 2 & -1 \\
0 & & -1 & 1
\end{array}\right] .
$$

For the later analysis of the influence of the transmitted error $e$ on the position and velocity errors $x$ the following Lemma is helpful.

Lemma 1: Suppose $A_{S B}$ is given as in (9) with $b>0$ and $k>\frac{1}{4} \lambda_{\max }\left(L_{g}\right) b^{2}$, where $L_{g}$ is the grounded Laplacian of the symmetric bidirectional communication graph (10) and $\lambda_{\max }(\cdot)$ denotes the eigenvalue with the largest real part. Denote the least stable eigenvalue as $\lambda_{1}\left(A_{S B}\right)$. Then, for all vectors $v \in \mathbb{R}^{2 N}$ and all $t \geq 0$ the inequality $\left\|e^{A_{S B} t} v\right\| \leq$ $e^{\operatorname{Re}\left(\lambda_{1}\left(A_{S B}\right)\right) t} c_{V_{S B}}\|v\|$ holds.

Proof: By applying Theorem 1 and 2 from [9] on $L_{g}\left(\mathcal{G}_{S B}\right)$ and $A_{S B}$ we conclude that the $2 N$ eigenvalues of $A_{S B}, s_{l_{1,2}}\left(A_{S B}\right)$ with $l \in\{1, \ldots, N\}$ satisfy $s_{l_{1,2}}=$ $-\frac{\lambda_{l}\left(L_{g}\right) b}{2}\left(1 \pm \sqrt{1-\frac{4 k}{\lambda_{l}\left(L_{g}\right) b^{2}}}\right)$. With $k>\frac{1}{4} \lambda_{\max }\left(L_{g}\right) b^{2}$ we have $N$ different complex conjugated pairs of eigenvalues $s_{l_{1,2}}=-\frac{\lambda_{l}\left(L_{g}\right) b}{2} \pm \Im$ where $\Im$ is the imaginary part of $s_{l_{1,2}}$ and $\operatorname{Re}\left(s_{j}\right)<0 \forall j \in\{1, \ldots, 2 N\}$. Thus $A_{S B}$ is diagonalizable with the orthogonal matrix
$V_{S B}=\left[v_{1_{1}}, v_{1_{2}}, \ldots, v_{N, 2}\right]$, i.e., $A_{S B}=V_{S B} D_{S B} V_{S B}^{-1}$, where $v_{l_{i}}$ is the eigenvector corresponding to the eigenvalue $s_{l_{i}}$. Hence with $\left\|V_{S B}\right\|\left\|V_{S B}^{-1}\right\|=c_{V_{S B}}$ we can compute $\left\|e^{A_{S B} t} v\right\|=\left\|e^{V_{S B} D_{S B} V_{S B}^{-1} t} v\right\| \leq\left\|e^{D_{S B} t}\right\| c_{V_{S B}}\|v\| \leq$ $e^{\operatorname{Re}\left(\lambda_{1}\left(A_{S B}\right)\right) t} c_{V_{S B}}\|v\|$.

The foregoing Lemma can be seen as a counterpart to Lemma 5.1 in [2]. Now we can state our first main result.

Theorem 2: Assume the vehicle platoon of double integrator agents (1) with linear symmetric bidirectional eventbased control architecture, $b>0, k>\frac{1}{4} \lambda_{\max }\left(L_{g}\right) b^{2}$, combined with the trigger function (5) with $c_{0}, c_{1} \geq 0$, $c_{0}+c_{1}>0$ and $0<\alpha<\left|\operatorname{Re}\left(\lambda_{1}\left(A_{S B}\right)\right)\right|$. Then $x(t)$ converges to a ball around the origin with radius $r_{S B}=c_{V_{S B}} \sqrt{N}\left\|B_{S B}\right\| \frac{c_{0}}{\left|\operatorname{Re}\left(\lambda_{1}\left(A_{S B}\right)\right)\right|}$ and Zeno behavior is excluded.

Proof: The proof follows similar steps as the proof of Theorem 5.2 in [2]. Firstly we compute an upper bound on the norm of the state error vector $\|x(t)\|=$ $\left\|e^{A_{S B} t} x(0)+\int_{0}^{t} e^{A_{S B}(t-s)} B_{S B} e(s) \mathrm{d} s\right\|$. Now we can apply Lemma 1 to compute $\|x(t)\| \leq e^{\operatorname{Re}\left(\lambda_{1}\left(A_{S B}\right)\right) t} c_{V_{S B}}\|x(0)\|+$ $\left\|B_{S B}\right\| \int_{0}^{t} e^{\operatorname{Re}\left(\lambda_{1}\left(A_{S B}\right)\right)(t-s)} c_{V_{S B}}\|e(s)\| \mathrm{d} s$. From (5) we derive $\|e(s)\| \leq \sqrt{N}\left(c_{0}+c_{1} e^{-\alpha s}\right)$. Using this bound and calculating the integral leads to $\|x(t)\| \leq e^{\operatorname{Re}\left(\lambda_{1}\left(A_{S B}\right)\right) t} c_{V_{S B}}\|x(0)\|+$ $\left\|B_{S B}\right\| c_{V_{S B}} \sqrt{N}\left(\frac{c_{1}}{\left|\operatorname{Re}\left(\lambda_{1}\left(A_{S B}\right)\right)+\alpha\right|} e^{-\alpha t}+\frac{c_{0}}{\left|\operatorname{Re}\left(\lambda_{1}\left(A_{S B}\right)\right)\right|}\right)$.

Under the given assumptions $\left(0<\alpha<\left|\operatorname{Re}\left(\lambda_{1}\left(A_{S B}\right)\right)\right|\right)$ we see that

$$
\lim _{t \rightarrow \infty}\|x(t)\| \leq\left\|B_{S B}\right\| c_{V_{S B}} \sqrt{N} \frac{c_{0}}{\left|\operatorname{Re}\left(\lambda_{1}\left(A_{S B}\right)\right)\right|}=r_{S B}
$$

and therefore $x(t)$ converges to a ball around the origin with radius $r_{S B}$.

The next step is to exclude Zeno behavior. Therefore it is the goal to give a lower bound on the time $\tau=t_{k+1}^{i}-t_{k}^{i}$ between two events. At time $t_{k}^{i}$ we know that $h_{i}\left(t_{k}^{i}\right)=0$ and therefore with (4), for $t_{k}^{i} \leq t<t_{k+1}^{i}$, we have

$$
\left\|h_{i}(t)\right\| \leq \int_{t_{k}^{i}}^{t}\left\|\left[\begin{array}{c}
\dot{e}_{i}(s) \\
\dot{e}_{d_{i}}(s)
\end{array}\right]\right\| \mathrm{d} s=\int_{t_{k}^{i}}^{t}\left\|\left[\begin{array}{c}
e_{d_{i}}(s) \\
-u_{i}(s)
\end{array}\right]\right\| \mathrm{d} s .
$$

Using (5) we compute $\left\|\dot{h}_{i}(t)\right\| \leq\left|e_{d_{i}}(t)\right|+\left|u_{i}(t)\right| \leq$ $c_{0}+c_{1} e^{-\alpha t}+\left|u_{i}(t)\right|$, where $\left|u_{i}(t)\right|=\mid-k\left(-\tilde{p}_{i-1}+\right.$ $\left.2 \tilde{p}_{i}-\tilde{p}_{i+1}-e_{i-1}+2 e_{i}-e_{i+1}\right)-b\left(-\dot{\tilde{p}}_{i-1}+2 \dot{\tilde{p}}_{i}-\right.$ $\left.\dot{\tilde{p}}_{i+1}-e_{d_{i-1}}+2 e_{d_{i}}-e_{d_{i+1}}\right) \mid \leq 4(k+b)(\|x\|+$ $\left.\left\|h_{i}(t)\right\|\right)$. Using the bound on $\|x(t)\|$ from above we can compute $\left\|\dot{h}_{i}(t)\right\| \leq\left(c_{0}+c_{1} e^{-\alpha t}\right)(4(k+b)+1)+$ $\left(r_{S B}+k_{2} e^{-\alpha t}+k_{3} e^{\operatorname{Re}\left(\lambda_{1}\left(A_{S B}\right)\right) t}\right) 4(k+b)$ with $k_{2}=$ $\left\|B_{S B}\right\| c_{V_{S B}} \sqrt{N} \frac{c_{1}}{\left|\operatorname{Re}\left(\lambda_{1}\left(A_{S B}\right)\right)+\alpha\right|}$ and $k_{3}=c_{V_{S B}}\|x(0)\|$. In the following we will investigate two different cases, depending on $c_{0}$.

Case 1: $c_{0} \neq 0$

For $t_{k}^{i} \leq t<t_{k+1}^{i}$ we have $\left\|\dot{h}_{i}(t)\right\| \leq\left(c_{0}+c_{1}\right)+$ $\left(c_{0}+c_{1}+r_{S B}+k_{2}+k_{3}\right) 4(k+b)=: C_{1}$ and therefore we know $\left\|h_{i}(t)\right\| \leq C_{1}\left(t-t_{k}^{i}\right)$ and the next event will not be triggered before time $t^{*}$ fulfills $C_{1}\left(t^{*}-t_{k}^{i}\right)=C_{1} \tau_{\min }=c_{0} \leq$ 


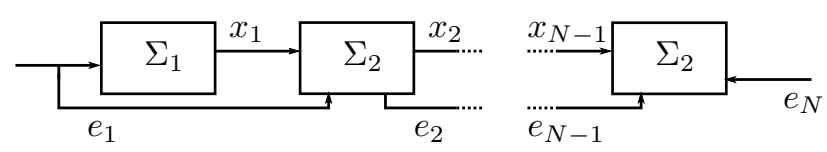

Fig. 2. Structure for Proposition 3

$c_{0}+c_{1} e^{-\alpha t}$. Hence we have a lower bound $\tau_{\min }=c_{0} / C_{1}$ on the time between two events and Zeno behavior is excluded in this case.

Case 2: $c_{0}=0$

Setting $c_{0}=0$ we compute the upper bound $\left\|\dot{h}_{i}(t)\right\| \leq\left(k_{2} e^{-\alpha t_{k}^{i}}+k_{3} e^{\operatorname{Re}\left(\lambda_{1}\left(A_{S B}\right)\right) t_{k}^{i}}\right) 4(k+b)+$ $c_{1} e^{-\alpha t_{k}^{2}}(4(k+b)+1)=: C_{1}\left(t_{k}^{i}\right)$ for $t_{k}^{i} \leq t<t_{k+1}^{i}$. Same computation as in case $1\left(C_{1}\left(t_{k}^{i}\right) \tau_{\min }=c_{1} e^{-\alpha t^{*}}\right)$ and multiplying this equation with $e^{\alpha t_{k}^{i}}$ leads to the equation

$$
\begin{aligned}
\left(\left(k_{2}+k_{3} e^{\alpha+\operatorname{Re}\left(\lambda_{1}\left(A_{S B}\right)\right) t_{k}^{i}}\right) 4(k+b)\right. & \\
\left.c_{1}(4(k+b)+1)+\right) \tau_{\min } & =c_{1} e^{-\alpha \tau_{\min }} .
\end{aligned}
$$

The left side of the equation is a linear function in $\tau_{\min }$ with finite gain since $\alpha+\operatorname{Re}\left(\lambda_{1}\left(A_{S B}\right)\right)<0$, while the right side exponentially decreases from $c_{1}$. Therefore there exists a lower bound $\tau_{\min }>0$ which solves this equation and Zeno behavior is excluded for the case $c_{0}=0, c_{1}>0$.

Observe that it is possible to set $c_{0}=0$ if $c_{1}>0$ and therefore the closed-loop system can be made asymptotically stable.

\section{NONLINEAR EVENT-TRIGGERED CONTROL IN PREDECESSOR-FOLLOWING ARCHITECTURE}

Due to [1] the predecessor-following control architecture works better if a nonlinear controller is applied. For that reason we give an analysis for the case that $f, g: \mathbb{R} \rightarrow \mathbb{R}$ are nonlinear. In this analysis we use the concepts of inputto-state stability (ISS), comparison functions (class $\mathcal{K}, \mathcal{K} \mathcal{L}$ ) and sector nonlinearities, which are defined in [13].

Firstly we state a Proposition that gives a bound on the states of an interconnection like in Fig. 2.

Proposition 3: Referring to Fig. 2 assume system $\Sigma_{1}$ is ISS with respect to input $e_{1}$ with class $\mathcal{K} \mathcal{L}$ function $\beta_{1}$ and class $\mathcal{K}$ function $\gamma_{1}$, i.e.,

$$
\left\|x_{1}(t)\right\| \leq \beta_{1}\left(\left\|x_{1}\left(t_{0}\right)\right\|, t-t_{0}\right)+\gamma_{1}\left(\sup _{t_{0} \leq \tau \leq t}\left\|e_{1}(\tau)\right\|\right)
$$

and $\Sigma_{2}$ is ISS with respect to its inputs $e_{i-1}, e_{i}$ and $x_{i-1}$, with $\beta_{2} \in \mathcal{K} \mathcal{L}, \gamma_{2}^{\left(e_{i}\right)}, \gamma_{2}^{\left(e_{i-1}\right)}, \gamma_{2}^{\left(x_{i-1}\right)} \in \mathcal{K}$ and $\gamma_{2}^{\left(x_{i-1}\right)}(r)$ being linear in $r$, i.e., $\gamma_{2}^{\left(x_{i-1}\right)}(r)=c_{\gamma} r$, for all $i=2, \ldots, N$.

If there is an interconnection of these systems like shown in Fig. 2, then for all $t>t_{0}$ it holds that

$$
\begin{aligned}
\left\|x_{j}(t)\right\| \leq & \beta_{2}\left(\left\|x_{j}\left(t_{0}\right)\right\|, t-t_{0}\right)+c_{\gamma}^{j-1} \beta_{1}\left(\left\|x_{1}\left(t_{0}\right)\right\|, 0\right) \\
& +\sum_{k=2}^{j-1} c_{\gamma}^{j-k} \beta_{2}\left(\left\|x_{k}\left(t_{0}\right)\right\|, 0\right)
\end{aligned}
$$

$$
\begin{aligned}
& +\left(c_{\gamma}^{j-1} \gamma_{1}+\sum_{l=0}^{j-2} c_{\gamma}^{l}\left(\gamma_{2}^{\left(e_{i}\right)}+\gamma_{2}^{\left(e_{i-1}\right)}\right)\right) \\
& \circ\left(\sup _{t_{0} \leq \tau \leq t}\left\|\left[\begin{array}{c}
e_{1} \\
\vdots \\
e_{j}
\end{array}\right](\tau)\right\|\right), \forall j \in\{2, \ldots, N\}
\end{aligned}
$$

Proof: The Proposition is proven by induction. Firstly we show, that (12) holds for $j=2$. Because $\Sigma_{2}$ is ISS we know that $\left\|x_{2}(t)\right\| \leq \beta_{2}\left(\left\|x_{2}\left(t_{0}\right)\right\|, t-t_{0}\right)+$ $\gamma_{2}^{\left(e_{i}\right)}\left(\sup _{t_{0} \leq \tau \leq t}\left\|e_{2}(\tau)\right\|\right)+\gamma_{2}^{\left(e_{i-1}\right)}\left(\sup _{t_{0} \leq \tau \leq t}\left\|e_{1}(\tau)\right\|\right)+$ $\gamma_{2}^{\left(x_{1}\right)}\left(\sup _{t_{0} \leq \tau \leq t}\left\|x_{1}(\tau)\right\|\right)$. Applying the bound from (11), using the linearity of $\gamma_{2}^{\left(x_{i-1}\right)}$ and the fact that $\beta_{1} \in$ $\mathcal{K} \mathcal{L}$ and $\left\|e_{1}(t)\right\|,\left\|e_{2}(t)\right\| \leq\left\|\left[\begin{array}{ll}e_{1}(t) & e_{2}(t)\end{array}\right]^{T}\right\|$, we get $\left\|x_{2}(t)\right\| \leq \beta_{2}\left(\left\|x_{2}\left(t_{0}\right)\right\|, t-t_{0}\right)+c_{\gamma} \beta_{1}\left(\left\|x_{1}\left(t_{0}\right)\right\|, 0\right)+$ $\left(c_{\gamma} \gamma_{1}+\gamma_{2}^{\left(e_{i}\right)}+\gamma_{2}^{\left(e_{i-1}\right)}\right) \circ\left(\sup _{t_{0} \leq \tau \leq t}\left\|\left[\begin{array}{ll}e_{1}(\tau) & e_{2}(\tau)\end{array}\right]^{T}\right\|\right)$ and therefore (12) is true for $j=2$.

Assuming it is also true for $j>2$ it suffices then to show that it is also valid for $j+1$. By the assumptions we know that the ISS property for $\left\|x_{j+1}\right\|$ holds and we know the bound (12) holds for $\left\|x_{j}\right\|$. Hence similar computation to the previous case and some index changes lead to

$$
\begin{aligned}
\left\|x_{j+1}(t)\right\| \leq & \beta_{2}\left(\left\|x_{j+1}\left(t_{0}\right)\right\|, t-t_{0}\right)+c_{\gamma}^{j} \beta_{1}\left(\left\|x_{1}\left(t_{0}\right)\right\|, 0\right) \\
& +\sum_{k=2}^{j} c_{\gamma}^{j+1-k} \beta_{2}\left(\left\|x_{k}\left(t_{0}\right)\right\|, 0\right) \\
& +\left(c_{\gamma}^{j} \gamma_{1}+\sum_{l=0}^{j-1} c_{\gamma}^{l}\left(\gamma_{2}^{\left(e_{i}\right)}+\gamma_{2}^{\left(e_{i-1}\right)}\right)\right) \\
& \circ\left(\sup _{t_{0} \leq \tau \leq t}\left\|\left[\begin{array}{lll}
e_{1} & \ldots & e_{j+1}
\end{array}\right]^{T}(\tau)\right\|\right) .
\end{aligned}
$$

This shows that (12) holds for $j+1$ under the assumption that it holds for $j>2$. Thus the induction step is verified and the Proposition holds for all $j \in\{2, \ldots, N\}$.

The next step is to give a Lemma which helps to compute the ISS gains for $\Sigma_{1}$ and $\Sigma_{2}$.

Lemma 4: Assume that $f, g$ are globally Lipschitz with Lipschitz constants $L_{1}, L_{2}$ and that they fulfill the following sector nonlinearities, $f \in\left[\epsilon_{1}, K_{1}\right]$ and $g \in\left[\epsilon_{2}, K_{2}\right]$, where $\epsilon_{1}, \epsilon_{2}, K_{1}, K_{2}>0$. Then the system

$$
\begin{aligned}
& \dot{y}_{1}=y_{2} \\
& \dot{y}_{2}=-f\left(y_{1}-u_{1}\right)-g\left(y_{2}-u_{2}\right)
\end{aligned}
$$

is ISS with respect to $u=\left[\begin{array}{ll}u_{1} & u_{2}\end{array}\right]^{T}$ with $\gamma^{(u)}(r)=c_{\gamma} r$. The value for $c_{\gamma}$ can be computed as

$$
c_{\gamma}=\sqrt{\frac{\lambda_{\max }(P)+\eta K_{1}}{2 \lambda_{\min }(P)}} \frac{4 \eta \max \left\{L_{1}, L_{2}\right\}}{\theta \min \left\{\epsilon_{1},\left(\eta \epsilon_{2}-1\right)\right\}},
$$

with $\eta>\max \left\{1, \frac{1}{\epsilon_{2}}+\frac{\left(1+K_{2}\right)^{2}}{\epsilon_{1} \epsilon_{2}}\right\}, P=\left[\begin{array}{cc}1 & 1 \\ 1 & \eta\end{array}\right], 0<\theta<1$ and $\lambda_{\min / \max }(P)$ refers to the smallest/largest eigenvalue of $P$.

Proof: From Proposition 1 in [1] it is known that system (13) is ISS. However, there is no computation for the ISS gain. Nevertheless we can use the Lyapunov function 
given in [1], $V(y)=\frac{1}{2} y^{T} P y+\eta \int_{0}^{y_{1}} f(z) \mathrm{d} z$, with $\eta>$ $\max \left\{1, \frac{1}{\epsilon_{2}}+\frac{\left(1+K_{2}\right)^{2}}{\epsilon_{1} \epsilon_{2}}\right\}$ and $P=\left[\begin{array}{ll}1 & 1 \\ 1 & \eta\end{array}\right]$ to compute this gain. It is also shown, that $\alpha_{1}(\|y\|)=\lambda_{\min }(P)\|y\|^{2} \leq$ $V(y) \leq \frac{\lambda_{\max }(P)+\eta K_{1}}{2}\|y\|^{2}=\alpha_{2}(\|y\|)$. Now we compute the derivative of $V$ along trajectories of (13):

$$
\begin{aligned}
\dot{V}= & {\left[\begin{array}{ll}
y_{1}+y_{2} & y_{1}+\eta y_{2}
\end{array}\right]\left[\begin{array}{l}
\dot{y}_{1} \\
\dot{y}_{2}
\end{array}\right]+f\left(y_{1}\right) \dot{y}_{1} \eta } \\
= & \underbrace{-y_{1} f\left(y_{1}\right)-\eta y_{2} g\left(y_{2}\right)+y_{2}^{2}+y_{1} y_{2}-y_{1} g\left(y_{2}\right)}_{(*)} \\
& +\eta y_{2}\left(f\left(y_{1}\right)-f\left(y_{1}-u_{1}\right)+g\left(y_{2}\right)-g\left(y_{2}-u_{2}\right)\right) \\
& +y_{1}\left(f\left(y_{1}\right)-f\left(y_{1}-u_{1}\right)+g\left(y_{2}\right)-g\left(y_{2}-u_{2}\right)\right)
\end{aligned}
$$

From [1] we know that with our assumptions $(*) \leq$ $-\frac{1}{2} \min \left\{\epsilon_{1}, \eta \epsilon_{2}-1\right\}\|y\|^{2}$. We also use the global Lipschitz property to compute $\dot{V} \leq-\frac{1}{2} \min \left\{\epsilon_{1},\left(\eta \epsilon_{2}-1\right)\right\}\|y\|^{2}+$ $\underbrace{\left(\eta\left|y_{2}\right|+\left|y_{1}\right|\right)\left(L_{1}\left|u_{1}\right|+L_{2}\left|u_{2}\right|\right)}_{(* *)}$. Further investigation yields $(* *) \leq \eta \max \left\{L_{1}, L_{2}\right\}\left\|\left[\begin{array}{ll}\left|y_{1}\right| & \left|y_{2}\right|\end{array}\right]\left[\begin{array}{ll}\left|u_{1}\right| & \left|u_{2}\right|\end{array}\right]^{T}\right\|+$ $\eta \max \left\{L_{1}, L_{2}\right\}\left\|\left[\begin{array}{ll}\left|y_{1}\right| & \left|y_{2}\right|\end{array}\right]\left[\begin{array}{ll}\left|u_{2}\right| & \left|u_{1}\right|\end{array}\right]^{T}\right\| . \quad$ Applying Hoelder's inequality with $p=q=2$ to compute $\dot{V} \leq$ $-\underbrace{\frac{1}{2}(1-\theta) \min \left\{\epsilon_{1},\left(\eta \epsilon_{2}-1\right)\right\}\|y\|^{2}}_{W_{3}(y)}-\frac{1}{2} \theta \min \left\{\epsilon_{1},\left(\eta \epsilon_{2}\right.\right.$

1) $\}\|y\|^{2}+2 \eta \max \left\{L_{1}, L_{2}\right\}\|u\|\|y\|$ with $0<\theta<1$. Thus $\dot{V} \leq-W_{3}(y)$ for all $\|y\| \geq \frac{4 \eta \max \left\{L_{1}, L_{2}\right\}}{\theta \min \left\{\epsilon_{1},\left(\eta \epsilon_{2}-1\right)\right\}}\|u\|=: \rho\|u\|$ with $W_{3}(y)$ being positive definite. Therefore we can apply Theorem 4.19 from [13] to show that system (13) is ISS with $\gamma^{(u)}(r)=\alpha_{1}^{-1} \circ \alpha_{2} \circ \rho(r)=$ $\sqrt{\frac{\lambda_{\max }(P)+\eta K_{1}}{2 \lambda_{\min }(P)}} \frac{4 \eta \max \left\{L_{1}, L_{2}\right\}}{\theta \min \left\{\epsilon_{1},\left(\eta \epsilon_{2}-1\right)\right\}} r=c_{\gamma} r$

Remark 5: If we extend system (13) with further inputs $v=\left[\begin{array}{ll}v_{1} & v_{2}\end{array}\right]^{T}$ and $w=\left[\begin{array}{ll}w_{1} & w_{2}\end{array}\right]^{T}$, such that

$\dot{y}_{1}=y_{2}$

$\dot{y}_{2}=-f\left(y_{1}-u_{1}+v_{1}-w_{1}\right)-g\left(y_{2}-u_{2}+v_{2}-w_{2}\right)$

the system is ISS with respect to $u, v$ and $w$ with $\gamma^{(u)}(r)=$ $\gamma^{(v)}(r)=\gamma^{(w)}(r)=c_{\gamma} r$.

Now we are able to state the main Theorem of this Section.

Theorem 6: Let the multi-agent system of double integrator agents (1) with predecessor-following event-based control architecture, where $f \in\left\{\epsilon_{1}, K_{1}\right\}$ and $g \in\left\{\epsilon_{2}, K_{2}\right\}$ are globally Lipschitz with constants $L_{1}, L_{2}$ combined with the trigger function (5) with $c_{0}>0, c_{1} \geq 0$ and $\alpha>0$. Then for all agents $j, x_{j}(t)=\left[\tilde{p}_{j}(t), \dot{\tilde{p}}_{j}(t)\right]$ is bounded by

$$
\begin{aligned}
\left\|x_{j}(t)\right\| \leq & \beta\left(\left\|x_{j}\left(t_{0}\right)\right\|, t-t_{0}\right)+\sum_{k=1}^{j-1} c_{\gamma}^{j-k} \beta\left(\left\|x_{k}\left(t_{0}\right)\right\|, 0\right) \\
& +\left(c_{\gamma}^{j}+2 \sum_{l=1}^{j-1} c_{\gamma}^{l}\right) \sqrt{j}\left(c_{0}+c_{1}\right), \forall t>t_{0}
\end{aligned}
$$

with $c_{\gamma}=\sqrt{\frac{\lambda_{\max }(P)+\eta K_{1}}{2 \lambda_{\min }(P)}} \frac{4 \eta \max \left\{L_{1}, L_{2}\right\}}{\theta \min \left\{\epsilon_{1},\left(\eta \epsilon_{2}-1\right)\right\}}$, where $\eta, P, \beta$ and $\theta$ are given in Lemma 4 and Zeno behavior is excluded.
Proof: The predecessor-following control scheme has the same structure as the one in Fig. 2. Additionally we get the ISS gains for $\Sigma_{1}$ and $\Sigma_{2}$ from Lemma 4 (and Remark 5) with $y=\left[\begin{array}{cc}\tilde{p}_{j} & \dot{\tilde{p}}_{j}\end{array}\right]^{T}, u=\left[\begin{array}{cc}\tilde{p}_{j-1} & \dot{\tilde{p}}_{j-1}\end{array}\right]^{T}$, $v=\left[\begin{array}{ll}e_{j} & e_{d_{j}}\end{array}\right]^{T}$ and $w=\left[\begin{array}{ll}e_{j-1} & e_{d_{j-1}}\end{array}\right]^{T}$. Therefore we can apply Proposition 3 to conclude that the norm of agent $j$ is bounded by (16) for all $t>t_{0}$.

The second part of the proof is to exclude Zeno behavior. We first try to give a bound on $\left\|\dot{h}_{j}\right\| \leq\left\|h_{j}\right\|+\left|u_{j}\right|$. Therefore we look at $\left|u_{j}(t)\right|$. Using the global Lipschitz property we derive the bound $\left|u_{j}(t)\right| \leq\left(L_{1}+L_{2}\right)\left(\left\|x_{j}\right\|+\right.$ $\left.\left\|x_{j-1}\right\|+\left\|h_{j}\right\|+\left\|h_{j-1}\right\|\right)$ and in combination with trigger law (5), the bound on the state and the assumption $c_{0}>0$ we conclude $\left\|\dot{h}_{j}\right\| \leq\left(1+2 L_{1}+2 L_{2}\right)\left(c_{0}+c_{1}\right)+\left(L_{1}+\right.$ $\left.L_{2}\right) \cdot\left(\beta\left(\left\|x_{j-1}\left(t_{0}\right)\right\|, 0\right)+\sum_{k=1}^{j-2} c_{\gamma}^{j-1-k} \beta\left(\left\|x_{k}\left(t_{0}\right)\right\|, 0\right)+\right.$ $\left(c_{\gamma}^{j-1}+2 \sum_{l=1}^{j-2} c_{\gamma}^{l}\right) \sqrt{j-1}\left(c_{0}+c_{1}\right)+\beta\left(\left\|x_{j}\left(t_{0}\right)\right\|, 0\right)+$ $\left.\sum_{k=1}^{j-1} c_{\gamma}^{j-k} \beta\left(\left\|x_{k}\left(t_{0}\right)\right\|, 0\right)+\left(c_{\gamma}^{j}+1 \sum_{l=1}^{j-1} c_{\gamma}^{l}\right) \sqrt{j}\left(c_{0}+c_{1}\right)\right)=:$ $C_{1}$. Hence similar arguments as in the proof of Theorem 2 leads to the bound $\tau_{\min } \geq \frac{c_{0}}{C_{1}}$ and therefore Zeno behavior is excluded since $c_{0}>0$ by assumption.

The following Corollary adds an asymptotic statement to the boundedness result from Theorem 6 .

Corollary 7: The nonlinear event-triggered predecessorfollowing controller has the converging input converging state (CICS) property, which is defined in [20], with respect to the input $e=\left[e_{1}, e_{d_{1}}, \ldots, e_{d_{N}}\right]^{T}$, i.e., if $e(t)$ converges to zero we know $\lim _{t \rightarrow \infty} x(t)=0$.

Proof: From [1] we know that the platoon with nonlinear predecessor-following controller is globally asymptotically stable with input $e=0$ (0-GAS). Boundedness of $\left\|x_{j}(t)\right\|$ for every $j$ is guaranteed through (16) and hence we conclude $\|x(t)\| \leq \sqrt{N} \max _{j}\left\|x_{j}(t)\right\|$ is bounded. Therefore the closed-loop system has the converging input bounded state property (CIBS) and we can derive the CICS property directly from the Proposition in [20].

\section{SIMULATION RESULTS}

In this Section we show simulation results for both presented controllers. We simulate a platoon with five cars, where the desired velocity is 1 and the desired distance between each car is 1 . The vehicles initially stand in the desired spacing. For the controller from Section III the parameters $k=1.84, b=1.4$, resulting in $\left|\operatorname{Re}\left(\lambda_{1}\left(A_{S B}\right)\right)\right|=$ 0.0567 as well as $c_{0}=10^{-4}, c_{1}=1$ and $\alpha=0.0561$ are chosen. Therefore Theorem 2 guarantees convergence to a ball around the origin with radius $r_{S B}=0.7197$. In Fig. 3 one can see how the norm of $x(t)$ evolves and which agent broadcasts its state at which time instant. The average time between two broadcasts in this simulation can be computed as $\tau_{\mathrm{avg}}=0.995 \mathrm{~s}$. If we simulate the platoon with the same controller and trigger the state information of each agent after a constant time $\tau_{c}$ the system is unstable already with $\tau_{c}=0.33 \mathrm{~s}$. Therefore at least in this simulation the event-triggered controller leads to a significant decrease of network load. Now we investigate the nonlinear predecessorfollowing controller with $g(z)=\tanh (z)+0.01 z$ and $f(z)=$ 


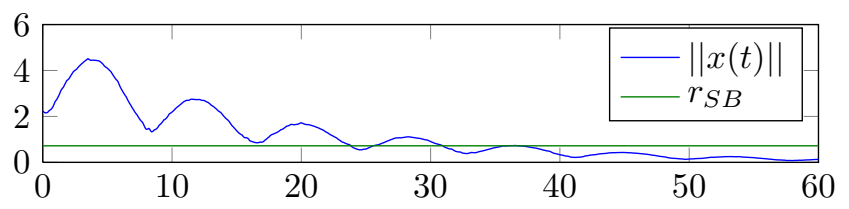

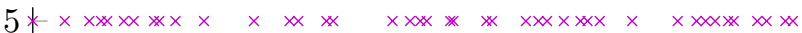

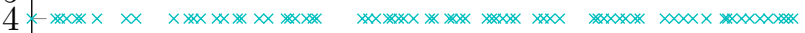

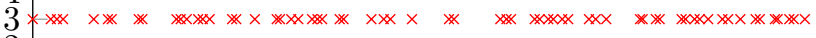

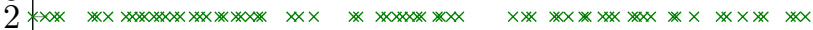

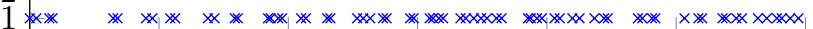
$\begin{array}{lllllll}0 & 10 & 20 & 30 & 40 & 50 & 60\end{array}$

time $[\mathrm{s}]$

Fig. 3. Evolution of $\|x(t)\|$ and inter-event times with event-triggered linear symmetric bidirectional controller
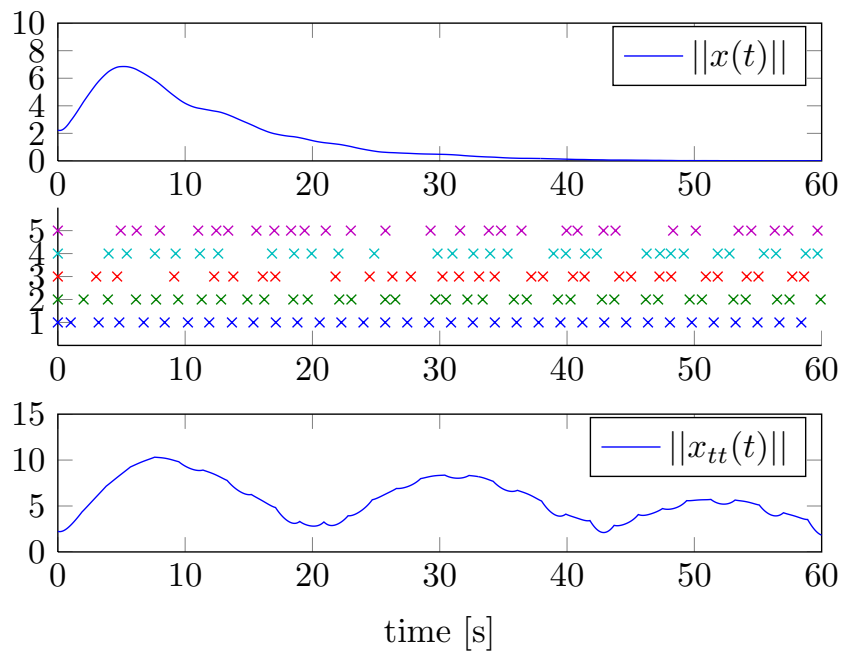

Fig. 4. Evolution of $\|x(t)\|$ with event- and time-triggered nonlinear predecessor-following controller and inter-event times

$0.1 g(z)$. In this case the conditions of Theorem 6 are satisfied with $L_{2}=K_{2}=1.01, \epsilon_{2}=0.01, L_{1}=K_{1}=0.1 L_{2}$ and $\epsilon_{1}=0.1 \epsilon_{2}$. Since we use the trigger rule with $c_{0}=10^{-4}$, $c_{1}=1$ and $\alpha=0.08$ the boundedness of the state error $x$ is guaranteed. The evolution of the state is shown in the upper part of Fig. 4. We see that $\|x(t)\|$ is not only bounded, it also converges to 0 . In this simulation we compute $\tau_{\text {avg }}=1.910 \mathrm{~s}$. As a comparison we simulate the same platoon with a constant exchange of information every $1.9 \mathrm{~s}$. One can see that the performance is worse in this case in the lower subplot of Fig. 4.

\section{CONCLUSIONS}

We proposed two event-triggered controllers for platooning. The linear symmetric bidirectional controller is shown to achieve convergence to a ball around the origin, which can be made arbitrarily small while Zeno behavior is still excluded. For the nonlinear predecessor-following controller we derived a bound on the norm of every agent's state and Zeno behavior is also excluded if the trigger rule has a positive offset. Furthermore we show that if the trigger error converges to zero, then also the state error converges to zero. For both controllers simulation results show that it is possible to achieve an event-triggered controller with a good performance that decreases the network load compared to a time-triggered controller with the same performance.

\section{REFERENCES}

[1] H. Hao and P. Barooah, "Stability and robustness of large platoons of vehicles with double-integrator models and nearest neighbor interaction," International Journal of Robust and Nonlinear Control, vol. 23, pp. 2097-2122, 2013.

[2] G. S. Seyboth, D. V. Dimarogonas, and K. H. Johansson, "Event-based broadcasting for multi-agent average consensus," Automatica, vol. 49, pp. 245-252, 2013.

[3] S. E. Shladover, C. A. Desoer, J. K. Hedrick, M. Tomizuka, J. Walrand, W.-B. Zhang, D. H. McMahon, H. Peng, S. Sheikholeslam, and N. McKeown, "Automatic vehicle control developments in the path program," IEEE Trans. Veh. Technol., vol. 40, no. 1, pp. 114-130, feb 1991.

[4] D. Swaroop and J. K. Hedrick, "String stability of interconnected systems," IEEE Trans. Automat. Contr., vol. 41, no. 3, pp. 349-357, mar 1996.

[5] Y. Zhang, E. B. Kosmatopoulos, P. A. Ioannou, and C. C. Chien, "Autonomous intelligent cruise control using front and back information for tight vehicle following maneuvers," IEEE Trans. Veh. Technol., vol. 48, no. 1, pp. 319-328, jan 1999.

[6] P. Seiler, A. Pant, and K. Hedrick, "Disturbance propagation in vehicle strings," IEEE Trans. Automat. Contr., vol. 49, no. 10, pp. 1835-1842, oct 2004

[7] P. Barooah and J. P. Hespanha, "Error amplification and disturbance propagation in vehicle strings with decentralized linear control," in Proc. IEEE Conf. Decision Control 44, Seville, Spain, dec 2005, pp. 4964-4969.

[8] S. Öncü, N. van de Wouw, W. P. M. H. Heemels, and H. Nijmeijer, "String stability of interconnected vehicles under communication constraints." in Proc. IEEE Conf. Decision Control 51, Maui, HI, dec 2012, pp. 2459-2464.

[9] H. Hao, P. Barooah, and J. J. P. Veerman, "Effect of network structure on the stability margin of large vehicle formation with distributed control," in Proc. IEEE Conf. Decision Control 49, Atlanta, GA, dec 2010, pp. 4783-4788.

[10] H. Hao, P. Barooah, and P. G. Mehta, "Stability margin scaling laws for distributed formation control as a function of network structure," IEEE Trans. Automat. Contr., vol. 56, no. 4, pp. 923-929, apr 2011.

[11] K.-E. Årzén, "A simple event-based pid controller," in Proc. 14th IFAC World Congress, 1999, pp. 423-428.

[12] P. Tabuada, "Event-triggered real-time scheduling of stabilizing control tasks," IEEE Trans. Automat. Contr., vol. 52, no. 9, pp. 1680-1685, sep 2007.

[13] H. K. Khalil, Nonlinear Systems. Upper Saddle River, NJ: PrenticeHall, 2002.

[14] R. Postoyan, A. Anta, D. Nesic, and P. Tabuada, "A unifying lyapunovbased framework for the event-triggered control of nonlinear systems," in Proc. IEEE Conf. Decision Control and European Control Conference 50, Orlando, FL, dec 2011, pp. 2559-2564.

[15] X. Wang and M. D. Lemmon, "Event-triggering in distributed networked control systems," IEEE Trans. Automat. Contr., vol. 56, no. 3 , pp. 586-601, mar 2011.

[16] D. V. Dimarogonas, E. Frazzoli, and K. H. Johansson, "Distributed event-triggered control for multi-agent systems," IEEE Trans. Automat. Contr., vol. 57, no. 5, pp. 1291-1297, may 2012.

[17] R. Olfati-Saber and R. M. Murray, "Consensus problems in networks of agents with switching topology and time-delays," IEEE Trans. Automat. Contr., vol. 49, no. 9, pp. 1520-1533, 2004.

[18] C. Godsil and G. F. Royle, Algebraic Graph Theory. Springer New York, 2001.

[19] K. H. Johansson, M. Egerstedt, J. Lygeros, and S. Sastry, "On the regularization of zeno hybrid automata," Systems \& Control Letters, vol. 38 , no. 3 , pp. $141-150,1999$.

[20] E. D. Sontag, "Remarks on stabilization and input-to-state stability," in Proc. IEEE Conf. Decision Control 28, Tampa, FL, dec 1989, pp. 1376-1378. 